Research Article

\title{
Mechanism of Baclofen Inhibiting the Proliferation and Metastasis of GBM by Regulating YAP
}

\author{
Lin Zhu, ${ }^{1,2}$ Juan Lu, ${ }^{3}$ Zhijun Bao, ${ }^{2}$ and Shiwen Guo $\mathbb{i D}^{1}$ \\ ${ }^{1}$ Department of Neurosurgery, The First Affiliated Hospital of Xi'an, Jiaotong University, Xi'an 710061, China \\ ${ }^{2}$ Department of Neurosurgery, Affiliated3201 Hospital of Xi'an Jiaotong University, Han Zhong 723000, China \\ ${ }^{3}$ School of Foreign Studies, Shaanxi University of Technology, Han Zhong 723000, China
}

Correspondence should be addressed to Shiwen Guo; zhr6962012@163.com

Received 30 October 2021; Accepted 27 November 2021; Published 29 December 2021

Academic Editor: Weiguo Li

Copyright (C) 2021 Lin Zhu et al. This is an open access article distributed under the Creative Commons Attribution License, which permits unrestricted use, distribution, and reproduction in any medium, provided the original work is properly cited.

\begin{abstract}
This study explores the effect of baclofen on the malignant phenotype of glioblastoma (GBM) and the growth of xenograft tumors and investigates the related mechanisms, aiming to reveal the effect of baclofen on the occurrence and development of GBM. The development of new therapeutic drugs for GBM lays a theoretical and experimental foundation. Research results show that baclofen could inhibit GBM cell proliferation and migration and promote GBM cell apoptosis; baclofen dose- and time-dependently could induce GBM cell YAP phosphorylation. YAP participated in the effect of baclofen on GBM cell proliferation and migration inhibition. Baclofen induced YAP phosphorylation in GBM cells through the GABABR2-Gs-Lats1/2 signaling pathway. Baclofen could inhibit the expression of survivin and Bcl2. Baclofen inhibits subcutaneous tumors by inducing YAP phosphorylation in vivo.
\end{abstract}

\section{Introduction}

Glioma is the most important and common central nervous system tumor and fatal primary brain tumor [1]. It is estimated that glioblastoma (GBM) accounts for $55 \%$ of all brain tumors [2]. Although radiation therapy, chemotherapy, and surgery have made great progress in the treatment of tumors, the prognosis of malignant GBM is still very poor, and the average survival time of patients is less than 1.5 years [3]. GBM is high malignant glioma. The molecular mechanism of GBM is a multistep development process of multigene changes [4]. In clinicopathology, the occurrence of GBM can be expressed in two different ways: primary and recurrent, suggesting that there may be some differences in molecular genetic changes between primary and recurrent GBM [5]. Previous efforts in GBM genome characterization identified GBM genes including growth factor receptors (EGFR, KIT, PDGFRA, FGFR1, FGFR3, and MET), mitogen-activated protein kinase (MAPK), and phosphoinositide 3-kinase (PI3K) signaling pathways, which includes PTEN, PIK3CA, NF1, BRAF, cell cycle pathways (MDM2, TP53,
CDK4/6, and RB1), and others (IDH1, MYC, and MYCN) [6]. With the further understanding of the molecular pathways driving malignant tumors in malignant GBM, various biomarkers and several drugs targeting specific molecular pathways in malignant cells have been developed. Despite the progress of current treatment, the prognosis of GBM is still poor, and the median survival time is only $12-15$ months $[7,8]$. In addition, in GBM, rapid tumor development and resistance to chemotherapy and radiotherapy are common, resulting in low 1-year survival. Therefore, further research is needed to explore the molecular mechanism of glioma progression and find new and effective glioma treatment strategies.

The Hippo pathway is an evolutionary conserved kinase cascade. The mammalian Hippo pathway consists of a dual kinase module, in which Mst1 and mst2 (Hippo homology in Drosophila) cooperate with their binding partner SAV1 to phosphorylate and activate Lats1/2. With the help of cofactor mob-1, they directly phosphorylate YAP and TAZ, resulting in cytoplasmic retention and proteomic degradation. In the "Hippo OFF" state, YAP/TAZ is set free of 
inhibitory phosphorylation [9]. When the upstream kinase is inactivated, dephosphorylated YAP/TAZ translocates to the nucleus, binds to TEAD1-4, and induces the expression of target genes [10]. Hippo signaling pathways play pivotal roles in governing organ size through balancing cell proliferation and apoptosis; moreover, accumulating evidence suggests that the Hippo pathway is dysregulated in GBM [11]. Studies have found that YAP-expressed GBM cells showed stronger tumorigenicity and caused enhanced tumor growth [12]. Nicholas Artinian et al. discovered that $\mathrm{Ser}^{760}$-phosphorylated AMOTL2 is incapable of binding YAP and leads to YAP-induced GBM cell migration and invasiveness [13]. Although the overexpression of YAP promotes the proliferation and invasion of GBM cancer cell lines, the role of YAP in the formation and progression of new cancers remain unclear. Understanding the molecular mechanism of heterogeneous tumor cell population in brain tumors may have a far-reaching impact on treatment management.

$\gamma$-Aminobutyric acid (GABA) is an important inhibitory neurotransmitter in the central nervous system. It has good water solubility and thermal stability. It has been proved that GABA, as a small molecular weight nonprotein amino acid, has edible safety and can be used in the production of beverages and other foods. Studies have shown that a certain amount of GABA can improve the body's sleep quality and reduce blood pressure. GABA acts on two different types of receptors and provides a powerful inhibitory effect based on their physiological and pharmacological properties. GABA type A (GABAA) receptor is a ligand-gated chloride channel which mediates fast inhibitory signals through rapid postsynaptic membrane hyperpolarization, whereas the metabotropic GABAB receptor produces slow and prolonged inhibitory signals via $G$ proteins and second messengers. $G A B A B$ receptors are members of class CG protein-coupled receptor (GPCR) family. GABAB receptors are prototypical heterodimers of R1 and R2 subunits; it is necessary for GABAB receptors to form R1/R2 heterodimers to produce GABAmediated GPCR functions. Recent studies suggest that the Hippo pathway is a downstream branch of GPCR signaling. Many GPCRs mediated signals can modulate YAP/TAZ activity depending on the type of downstream $G$ proteins [10]. However, GABA signaling remains extremely complex, and the multiple possible interaction of GABA (B) subunits with the Hippo pathway in GBM has not been well established. The mechanism of action of related proteins is shown in Figure 1.

Baclofen, ( \pm )-4-amino-3-(p-chlorophenyl)-butanoic acid, is a structural analog of GABA. Baclofen can cross the bloodbrain barrier via neutral amino acid transporters, acting as agonist of the GABAB receptor [14-16]. Baclofen was shown to reduce hepatocellular carcinoma, gastric, and colon cancer development in vitro and in vivo $[17,18]$, but its relationship with GMB has not yet been reported so far. In this study, we found that baclofen could inhibit the proliferation and migration of GBM cells, which reduce the invasiveness of GBM, in a dose- and time-dependent manner. Molecular mechanisms underlying the baclofen's effects are related to YAP of the Hippo pathway. What is more, baclofen induced phosphorylation of YAP in GBM cells through the GABABR1/2Gs-Lats1/2 signaling pathway, which leads to the degradation of YAP, and pYAP could not activate the downstream surviving genes such as survivin and $\mathrm{Bcl} 2$, and lower level of proliferation-associated genes may be the reason of tumor inhibition. This may explain baclofen GBM cancer inhibition mechanism.

\section{Methods}

2.1. Cell Culture and Transfection. U251 cells were purchased from Cell bank of representative culture preservation Committee of Chinese Academy of Sciences. U251 cells were cultured in DMEM (Dulbecco's modified Eagle's medium, ThermoFisher, Shanghai, China) supplemented with $10 \%$ FBS (fetal bovine serum, ThermoFisher, Shanghai, China) and cultured at $37^{\circ} \mathrm{C}$ in a $5 \% \mathrm{CO}_{2}$ incubator. GABABR2 shRNA plasmids (sc-42463-SH) were purchased from Santa Cruz; YAP downregulated and upregulated lentiviruses were constructed and obtained from GeneChem (Shanghai, China). The transfection process was carried out according to the manufacturer's guidance.

2.2. Proliferation Assay [19]. Cell Counting Kit-8 (APExBIO, USA) was purchased for detecting the cell proliferation rate. $1 \times 10^{3}$ treated cells were seeded in 96-well plates per well and cultured at $37^{\circ} \mathrm{C}$ and $5 \% \mathrm{CO}_{2}$ for 24 hours. Cells were cultured after 12, 24, and 48 hours, respectively, and incubated with CCK- 8 testing solution for 2 hours. Then, absorbance was measured at the wavelength of $450 \mathrm{~nm}$ to represent the cell proliferation ability.

2.3. Transwell Assay [19]. Cell suspension was prepared, and $1 \times 10^{5}$ cells/well were inoculated into the supraventricular cavity of Transwell. $100 \mu \mathrm{L}$ serum-free DMEM medium was added to the superior chamber: $15 \%$ fetal bovine serum and $600 \mu \mathrm{L}$ DMEM medium. The cells were routinely cultured for 24 hours. Then, the cells on the surface of the lower membrane were fixed with $4 \%$ paraformaldehyde and stained with crystal violet. The migrated cells were fixed and counted.

2.4. Western Blot. The total protein of GBM cells was extracted, and the protein concentration was measured. Make SDS-polyacrylamide gel to separate the protein and transfer it to PVDF membrane (Millipore, USA). 5\% nonfat milk was used to block the transferred PVDF membrane. Then, phospho-YAP $(1: 1000)$, YAP $(1: 1000)$, GABABR1 $(1: 500)$, anti-GABABR2 $(1: 2000)$, Lats1 $(1: 1000)$, Lats2 $(1: 1000)$, survivin $(1: 1000)$, and Bcl $2(1: 1000)$ primary antibodies were incubated overnight at $4^{\circ} \mathrm{C}$, and secondary antibodies (1: 10000) were incubated for 1 hour. ECL chemiluminescent kit and Quantity One software were used to detect the protein expression level.

2.5. Apoptosis [19]. FAM-FLICA ${ }^{\circledR}$ caspase-3/7 assay kit was used to detect the apoptosis rate. FLICA was added to each sample at 1:30 and incubated for 1 hour. Then, the fluorescence microscope was used to detect the caspase $3 / 7$ activity. FAM-FLICA excites at $492 \mathrm{~nm}$ and emits at $520 \mathrm{~nm}$. 


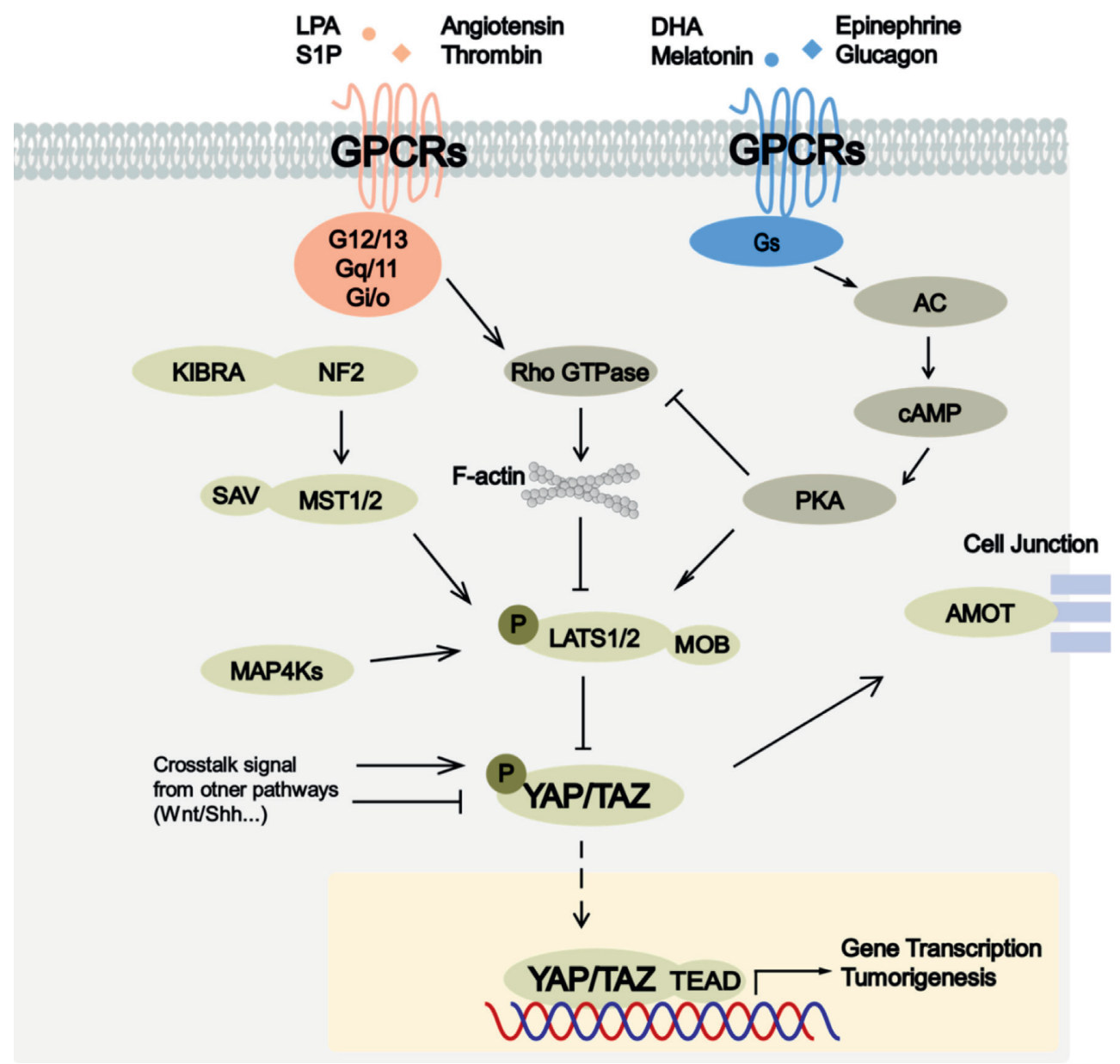

FIGURE 1: Diagram of the mechanism of protein action.

2.6. Animal Model. Ten four-week-old nude mice were recruited (18-22 g, Beijing Weitong Lihua Laboratory Animal Technology Co., Ltd.). We used $2 \times 10^{6}$ U251 cells to subcutaneously inject into the left flank of the nude mice. Mice were randomly classified into the control group $(0.9 \%$ $\mathrm{NaCl}$ injected from tail vein) and baclofen treatment group (100 $\mu \mathrm{M}$ baclofen injected from tail vein). Thirty days later, the tumor was collected, and the changes of various proteins in the tumor tissue were detected. Tumor volume was calculated as $0.5 \times$ length $\times$ width $^{2}$ every third day.

2.7. Statistics. The statistics analysis was conducted, and figures were graphed using GraphPad Prism 8.2. Student's $t$-test was used to evaluate the differences between two groups. One-way ANOVA was used to investigate the statistical significance among multiple groups. $P<0.05$ was thought to be statistically significant.

\section{Results}

3.1. Baclofen Inhibited Proliferation and Migration and Promoted Apoptosis of GBM Cells. In order to investigate the function of baclofen, we treated GBM cells (U251) with 10, 50,100 , and $120 \mu \mathrm{M}$ baclofen for 18 hours. After that, we found that $100 \mu \mathrm{M}$ or more baclofen could significantly suppress the viability of U251 (Figure 2(a)). Moreover, baclofen could inhibit the migration of and promote the apoptosis of GBM cells (Figures 2(b) and 2(c)).

3.2. Baclofen Dose- and Time-Dependently Induced Phosphorylation of YAP in GBM Cells. We treated U251 cell line with $10,50,100$, and $120 \mu \mathrm{M}$ baclofen, respectively. The results turned out that YAP phosphorylation was induced when treated with $50 \mu \mathrm{M}$ baclofen; besides, $100 \mu \mathrm{M}$ baclofen was the optimal concentration in inducing the phosphorylation of YAP (Figure 3(a)). Furthermore, we used $100 \mu \mathrm{M}$ baclofen to treat U251 cell line for 12, 18, 24, and 36 hours and found that the phosphorylation of YAP was induced on the condition that baclofen was incubated with U251 cell line for at least 18 hours (Figure 3(b)).

3.3. YAP Involved in Baclofen-Induced Inhibition of Proliferation and Migration of GBM Cells. After downregulating the expression of YAP, the proliferation and migration ability of U251 were significantly suppressed (Figures 4(a) and $4(\mathrm{~b}))$. Besides, the overexpression of YAP resulted in increased proliferation and migration rate (Figures 4 (c) and $4(d))$. We then increased the YAP expression in baclofenincubated U251 cell line, and we noticed that baclofen- 


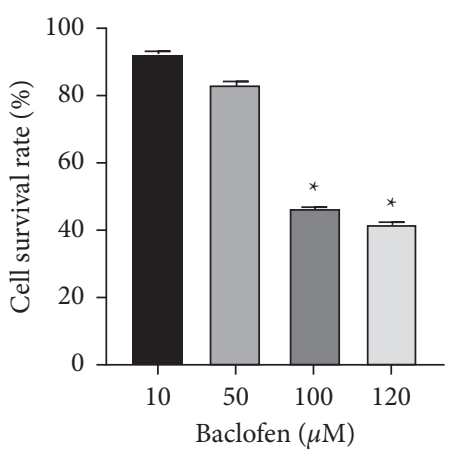

(a)

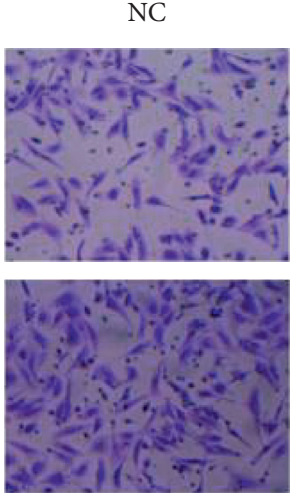

(b)

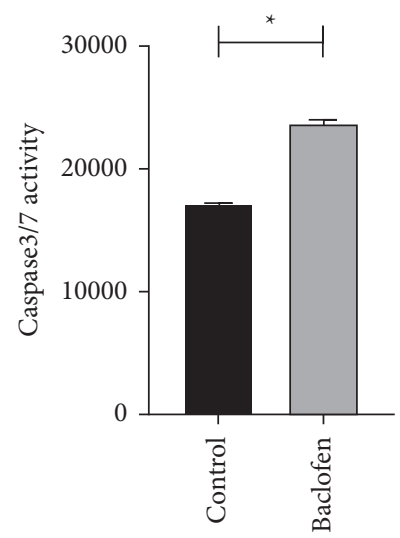

(c)

FIGURE 2: Baclofen inhibited proliferation and migration and promoted apoptosis of GBM cells. * Statistical significance compared with the control group.

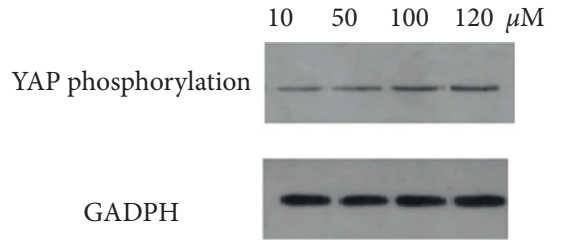

(a)

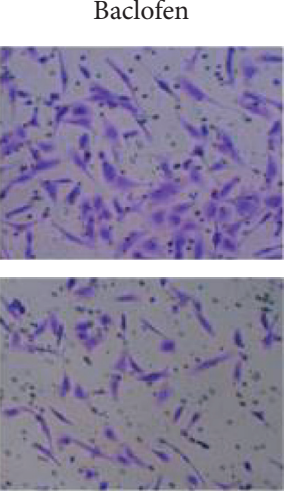

FIGURE 3: Baclofen dose and time-dependently induced phosphorylation of YAP in GBM cells.

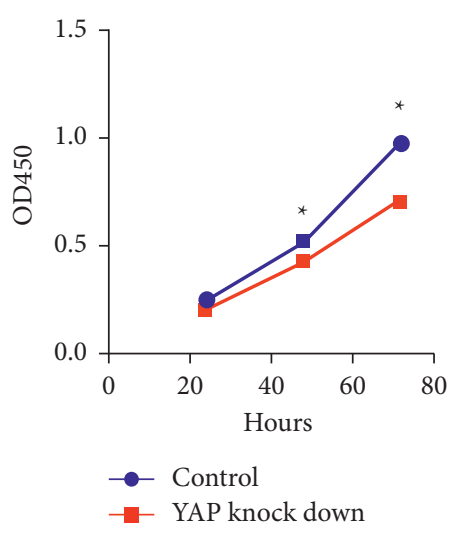

(a)

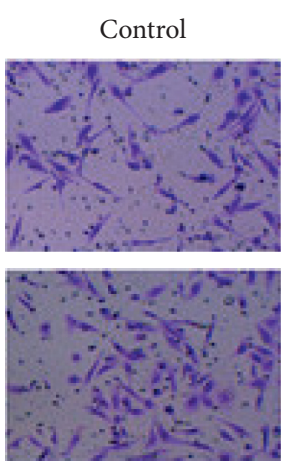

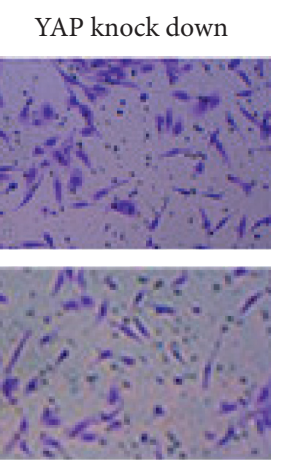

b)

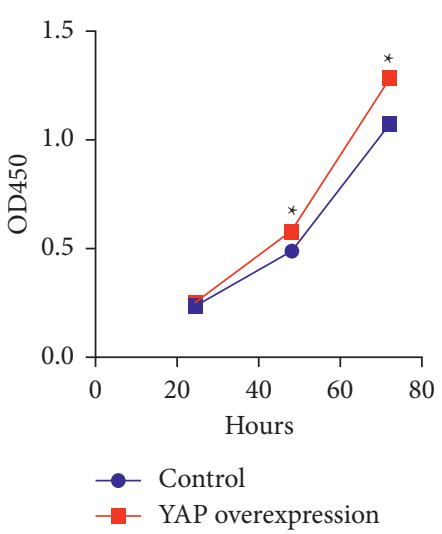

(c)

FIgUre 4: Continued. 

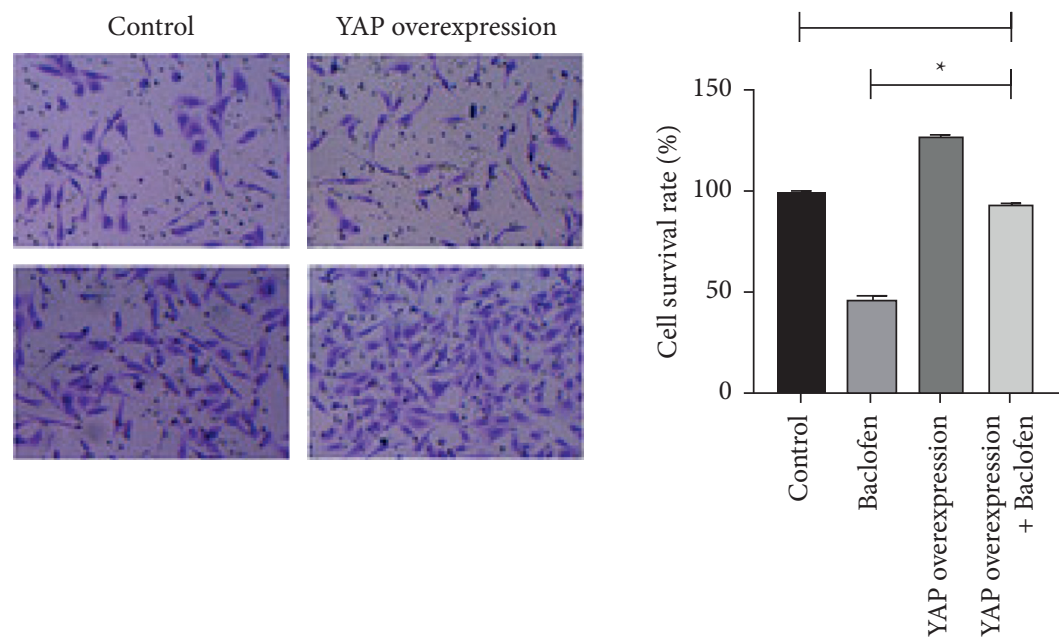

(d)

(e)

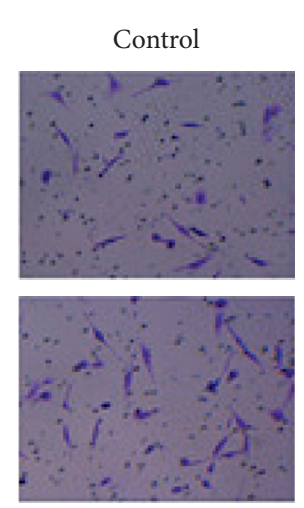

YAP overexpression + Baclofen
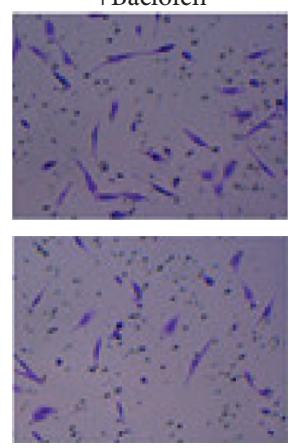

(f)

FIGURE 4: YAP involved in baclofen-induced inhibition of proliferation and migration of GBM cells. * Statistical significance compared with the control group.

induced inhibition of cell viability was significantly suppressed (Figure 4(e)). Moreover, Transwell assay showed that the baclofen-related suppression on migration ability was recused by YAP overexpression as well (Figure 4(f)).

\subsection{Baclofen-Induced Phosphorylation of YAP in GBM Cells} through GABABR2-Gs-Lats1/2 Signaling Pathway. We found that CGP 35348, a GABAB receptor antagonist, could reverse the baclofen-induced inhibition of proliferation of GMB cells; however, SR 95531 hydrobromide, a GABAB receptor antagonist showed a limited effect on baclofen-mediated proliferation inhibition (Figures 5(a) and $5(\mathrm{~b}))$. After treated with baclofen, we detected the significant increase in the expression of GABABR2 and Lats1; however, the expression of Lats2 was not significantly influenced (Figure 5(c)). After downregulating the expression of GABABR1 and GABABR2, respectively, we found that only GABABR2 knock down reversed the baclofen-induced inhibition of proliferation of GMB cells, suggesting that it is GABABR2 not GABABR1 that acted as the potential downstream target of baclofen (Figures 5(d) and 5(e)). Furthermore, we detected an increased expression of cAMP in U251 after treating with baclofen, indicating that GABABR2-Gs might be activated (Figure 5(f)).

3.5. Survivin and Bcl2 Acted as Downstream of YAP. We found that survivin and $\mathrm{Bcl} 2$ expression can be suppressed by baclofen treatment (Figure 6(a)). We assumed that survivin and $\mathrm{Bcl} 2$ were the downstream target of YAP. Therefore, we downregulated the expression of YAP and found that the expressions of survivin and $\mathrm{Bcl} 2$ were suppressed (Figure 6(b)); meanwhile, YAP overexpression led to upregulation of survivin and $\mathrm{Bcl} 2$ (Figure 6(c)).

3.6. Baclofen Inhibited Subcutaneous Tumors Generated by Inducing YAP Phosphorylation In Vivo. We have recruited 10 mice and classified them into treatment (baclofen) and control group $(0.9 \% \mathrm{NaCl})$. We found that baclofen significantly decreased the tumor growth in vivo (Figures 7 (a) and $7(b))$.

3.7. Changes of Transplanted Tumor Tissues in Nude Mice after Baclofen Treatment. Compared with the control group, the expression level of phosphorylated YAP protein in the 


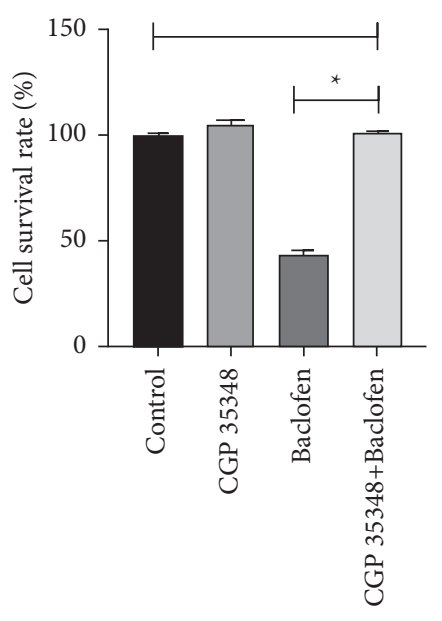

(a)

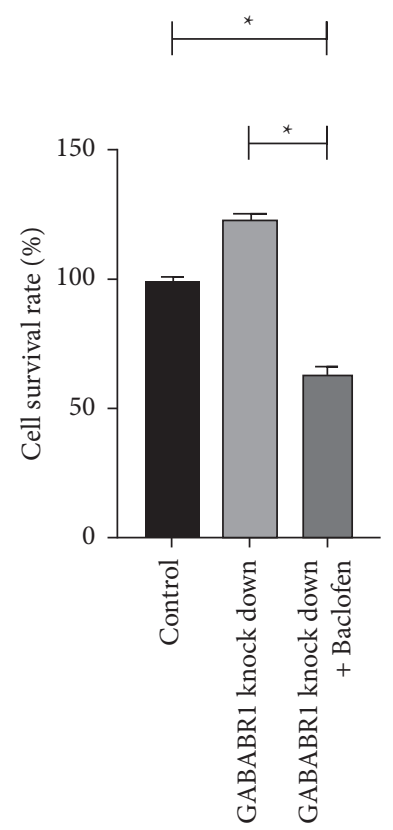

(d)

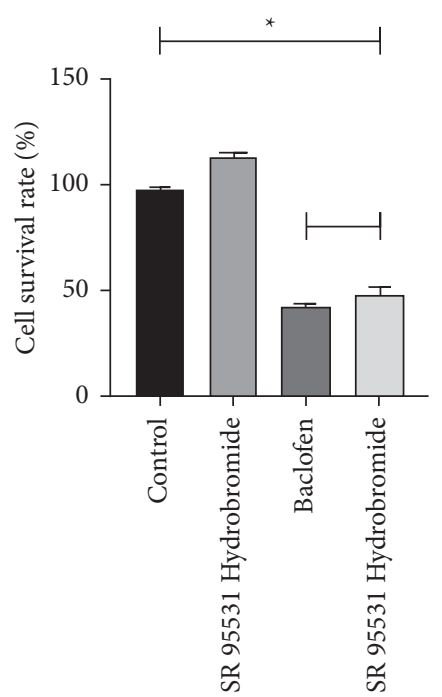

(b)

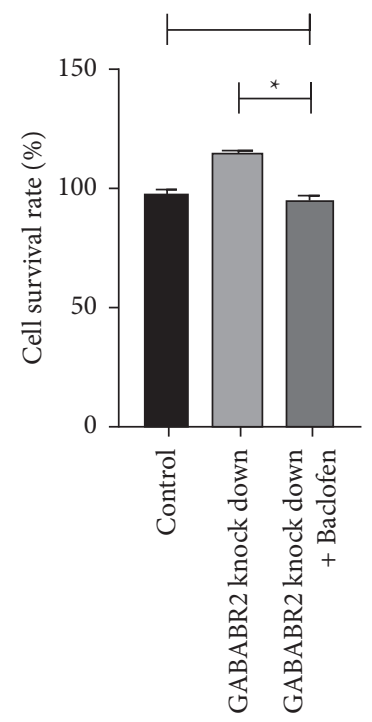

(c)

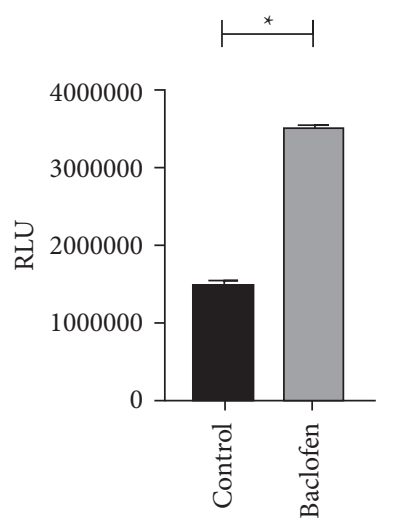

(e)

(f)

FIGURE 5: Baclofen-induced phosphorylation of YAP in GBM cells through the GABABR2-Gs-Lats1/2 signaling pathway. *Statistical significance compared with the control group.

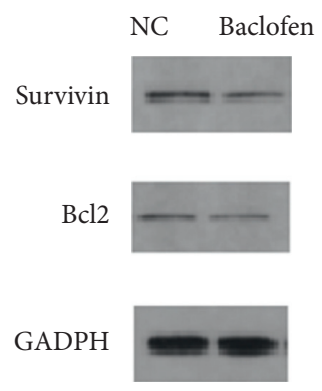

(a)
YAP

NC Knock down

Survivin

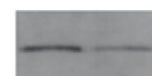

$\mathrm{Bcl} 2$

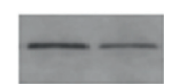

GADPH

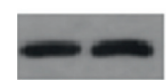

(b)
YAP

NC Overexpression

Survivin

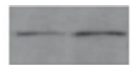

Bcl2

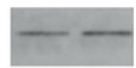

GADPH

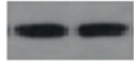

(c)

FIgURE 6: Survivin and Bcl2 acted as downstream of YAP. 


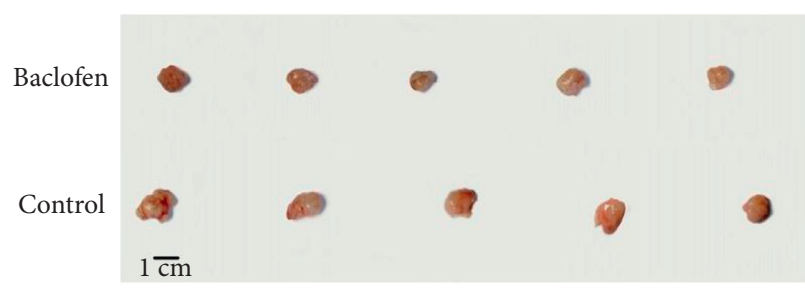

(a)

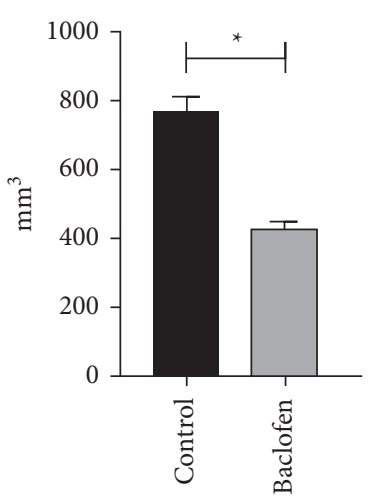

(b)

Figure 7: Baclofen inhibited subcutaneous tumors generated by inducing YAP phosphorylation in vivo. *Statistical significance compared with the control group.

transplanted tumor tissue of nude mice after the treatment of baclofen was significantly increased, indicating that the phosphorylation of YAP could also be induced by baclofen in vivo (Figure 8(a)). The expression levels of GABABR2 and Lats1 proteins in transplanted tumor tissues after the treatment of baclofen were significantly higher than those in the control group, indicating that baclofen can also promote the expression of GABABR2 and Lats 1 proteins in vivo (Figure 8(b)). The expression levels of survivin and $\mathrm{Bcl} 2$ proteins in transplanted tumor tissues after baclofen treatment were significantly lower than those in the control group, suggesting that survivin and $\mathrm{Bcl} 2$ proteins expression could also be inhibited by baclofen in vivo (Figure $8(\mathrm{c})$ ).

\section{Discussion}

GBM is the most frequent and most aggressive malignant primary brain tumor in adults, representing a highly heterogeneous group of neoplasms that are among the most aggressive and challenging cancers to treat. These tumors are typically associated with dismal prognosis and poor quality of life. It is the most frequent primary brain tumor, with a median overall survival (OS) between 10 and 20 months. High-grade gliomas are the leading cause of central nervous system (CNS) cancer-related death in both children and adults. Most people suffering from GBM, especially the elderly, have a lower survival within one year [4]. In GBM, the clinical trials of targeted therapy for most patients have a limited success rate, indicating that the current medical community still lacks understanding of the pathophysiology of gliomas.

Studies have shown that the GABAB signaling pathway participates in the occurrence of GBM through metabolic reprogramming and genetic transformation and accelerates the replication and progression of glioma. GABAB can upregulate cytoskeleton protein and intracellular $\mathrm{Ca}^{2+}$ level and increase the release of glutamate, so as to promote the formation of synaptic-like connection and the surrounding cell microenvironment [20]. GHB (4-hydroxybutyrate) exists in human brain and can act on
GABA receptor (GABABR). GABABR is mainly the main inhibitory neurotransmitter in the somatosensory system. Research has showed that GHB inhibits proliferation of GBM stem-like cells and alleviated tumor aggressiveness [21]. Therefore, GPCR is the main target of drugs at present, and the GPCR-Hippo signal pathway is a complex signal network. Drugs targeting GPCRs, G protein, or downstream signal nodes may affect effectors such as YAP/TAZ [10].

The main functions of YAP/TAZ include regulating cell proliferation and survival, maintaining the self-renewal of adult stem cells, participating in organ development, tissue size regulation, tissue regeneration, and so on. The Hippo signal pathway is a common YAP/TAZ upstream signal pathway, which was first found in the screening of tumor suppressor genes in Drosophila. Subsequently, the four core components of the signal pathway were revealed successively, including Wars, Salvador (SAV1/WW45), Hippo, and Mats. When the Hippo signaling pathway is turned on, YAP/ TAZ is phosphorylated by Lats $1 / 2$ at multiple sites, interacts with 14-3-3, and is retained in the cytoplasm; phosphorylation also leads to YAP/TAZ polyubiquitination and degradation [22]. When Hippo signaling is off, its dephosphorylated morphology could translocate into the nucleus to bind TEAD and VGLL4 to regulate target gene transcription. The functional mechanism of YAP/TAZ protein is shown in Figure 9.

Studies have shown that YAP phosphorylation can be mediated through relevant contact between cells, transmission of mechanical signals, induction of stress signals, cell polarity/structure, and cell cycle [23]. GPCR and Rho can promote YAP/TAZ nuclear localization and transcriptional activities. Stimulation of $\mathrm{G} \alpha$-coupled receptors by multiple ligands, including lysophospholipids sphingosine 1-phosphate (S1P), lysophosphatidic acid (LPA), thrombin, estrogens, and acetylcholine, results in YAP/TAZ activation through the promotion of a RhoGTPase-regulated F-actin cytoskeleton in a manner that is either Lats1/2dependent or independent. When tumors occur, the dysfunction of the Hippo signaling pathway can lead to the dephosphorylation and activation of YAP, which in turn 


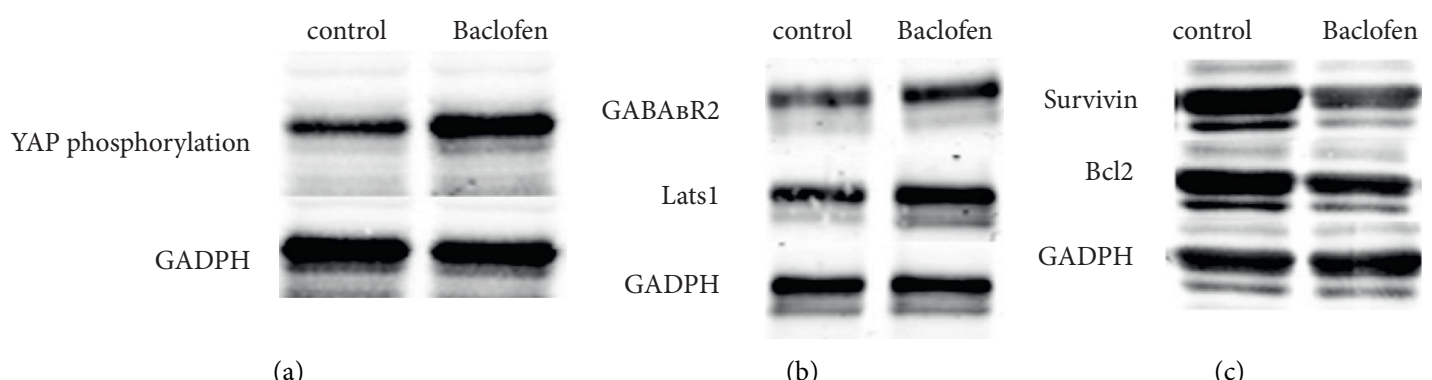

Figure 8: Changes of transplanted tumor tissues in nude mice after baclofen treatment.
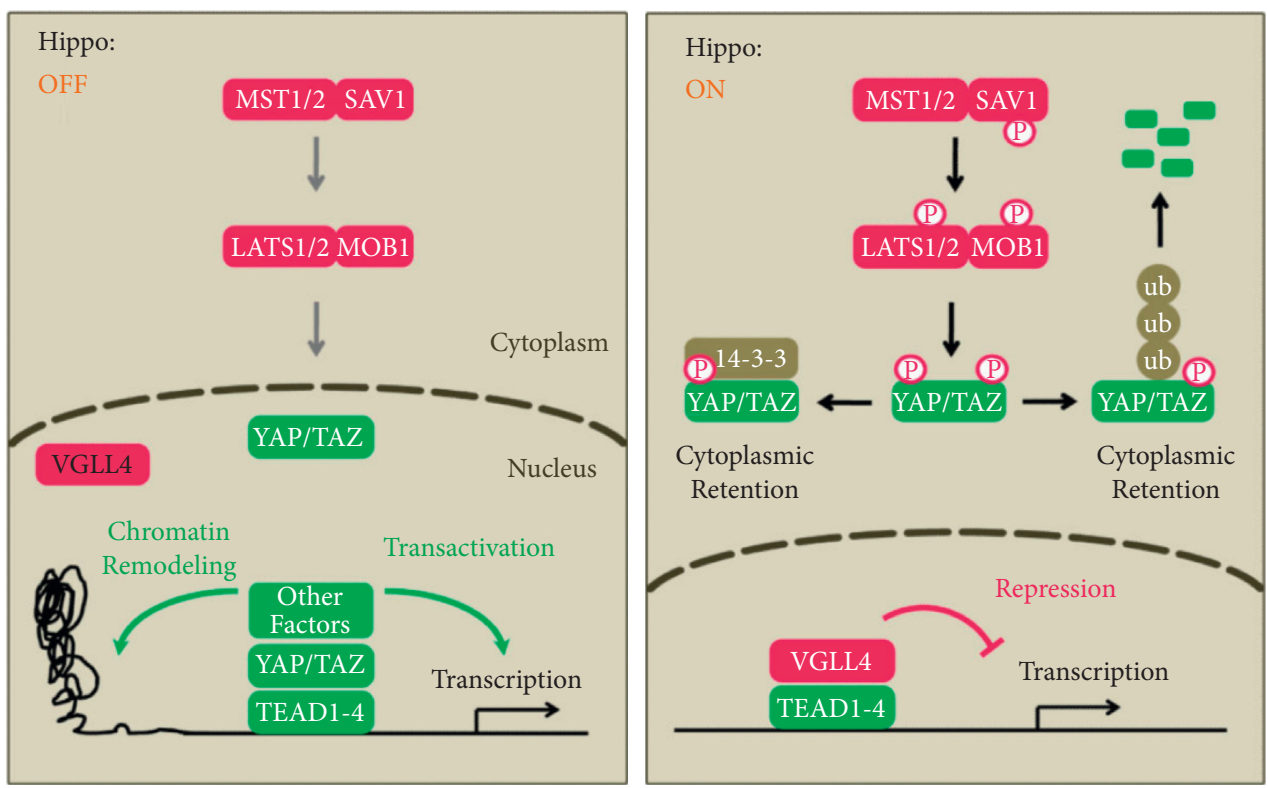

Hippo pathway components and major functions.

\begin{tabular}{|c|c|c|}
\hline Drosophila & Mammals & Major functions in Hippo pathway \\
\hline Hippo (Нpo) & MST1/2 & Phosphorylate LATS1/2, MOB1, and SAV1, leading to LATS1/2 activation \\
\hline Salvador (Sav) & SAV1 & Interacts with MST $1 / 2$, promotes phosphorylation of LATS $1 / 2$ by MST $1 / 2$ \\
\hline Warts (Wts) & LATS1/2 & Phosphorylate and inactivate YAP/TAZ \\
\hline Mats & $\mathrm{MOB} 1 \mathrm{~A} / \mathrm{B}$ & Scaffold protein of LATS1/2 \\
\hline Yorkie (Yki) & YAP/TAZ & Transcription co-activator, major effectors of the Hippo pathway \\
\hline Scalloped (Sd) & TEAD1-4 & Transcription factors mediate the effect of YAP/TAZ \\
\hline Tgi & VGLL4 & Competes with YAP/TAZ for TEADs, inhibits YAP/TAZ functions \\
\hline misshapen (Msn) & MAP4K4 & Activates LATS1/2 \\
\hline Merlin (Mer) & Merlin/NF2 & \multirow{3}{*}{$\begin{array}{c}\text { May form a complex and mediates upstream signals (from plasma } \\
\text { membrane) to MST1/2. NF2 may bring LATS1/2 to plasma membrane } \\
\text { and facilitate its activation by MST1/2 }\end{array}$} \\
\hline Kibra & KIBRA & \\
\hline \multirow[t]{2}{*}{ Expanded (Ex) } & FRMD6? & \\
\hline & AMOT & $\begin{array}{l}\text { Sequesters YAP/TAZ to cell junctions, binding and indirectly activates } \\
\text { LATS } 1 / 2 \text {; a substrate of LATS } 1 / 2\end{array}$ \\
\hline
\end{tabular}

FIGURE 9: Diagram of the functional mechanism of YAP/TAZ protein.

induces Ki67, c-Myc, SOX4, H19, AFP, BIRC5/survivin, BIRC2/cIAP1, and other cell proliferation-related genes, and apoptosis inhibits high expression [22].
Animal experiments show that YAP activation can cause tissue overgrowth, injury, and multiple organ lesions, resulting in tumor formation [24]. TAZ upregulation is 
detected in most GBMs, and high TAZ mRNA levels are associated with reduced survival. High levels of YAP expression are found in all levels of glioma subsets and are associated with shorter survival in glioma patients. SCID mice were injected with primary GBM cell line in situ, and TAZ gene knockout impaired tumor formation [11].

Baclofen has been used in treating this tumor in neuroscience for quite a time, but the precise mechanism of how baclofen inhibits GBM remains elusive. In this study, we have proved that baclofen can inhibit GBM cells invasion. The CCK- 8 test and Transwell test proved that baclofen can inhibit GMB cells' proliferation and migration. Baclofen could induce phosphorylation of YAP; as shown in Figure 9, the phosphorylated YAP retained in the cytoplasm and is degraded, leaving the downstream genes inactivated. Western blot analysis shows that the level of phosphorylated YAP is dose and time-dependent of baclofen. This may be the possible mechanism of baclofen in inhibiting the progression of GBM.

In most cases, soluble factors regulate YAP through their homologous $G$ protein-coupled receptors and related $G$ protein subunits through the Hippo pathway, so as to bind to small GTPase, RhoA, and rock, resulting in the change of Lats1/2 activation [23]. To determine which receptor was involved, siRNAs against the catalytic subunits of GABABR1/R2 and Gs and YAP upstream Lats1/2 were used. Western blot results show that baclofen-induced pYAP was reversed by these siRNAs, suggesting that YAP is activated by the GABABR1/2-Gs signaling pathway and is likely to be a direct substrate of Lats $1 / 2$.

We further examined the role of baclofen as a downstream effector in the expression of survivin and Bcl2. As shown in Figure 4, baclofen significantly decreased survivin and $\mathrm{Bcl} 2$ expression in both mRNA and protein levels, which means pYAP could not activate the transcription of genes that promote cellular survival, such as survivin and Bcl2, which means baclofen could inhibit tumor progression in affecting the expression of the survival genes, and lower level of proliferation-associated genes may be the reason of tumor-inhibition.

Bcl2 family members can counteract the BAK/BAX's ability to induce apoptosis; when in stress, $\mathrm{BH} 3$ members activate BAK/BAK, resulting in mitochondrial outer membrane permeabilization (MOMP). MOMP results in cytochrome $\mathrm{c}$ release, activation of downstream caspase, and finally cell death. During tumorigenesis, antiapoptotic Bcl2 is upregulated to make cancer cells survive. [25] The betacatenin YAP1-TBX5 complex transcriptionally upregulated Bcl2 family members, thus suppressing the mitochondrialinduced apoptosis pathway in cancer cells [26]. Other than Bcl2 family proteins, a second gene family called inhibitor of apoptosis protein (IAP) has been identified, which regulates various important aspects of cell survival. Survivin (encoded by BIRC5) is a member of the IAP family, which is overexpressed in most of the malignancies and totally absent in most of the normal tissues. Survivin has two main functions in cancer cells: (1) regulate mitosis by forming chromosome passenger complex (CPC) with other proteins and (2) inhibition of apoptosis by direct inhibition of active caspases
[27]. It is reported that survivin is a target of YAP because the level of survivin increases significantly when YAP is activated. Here, we determined the functional relationship between YAP and survivin in GBM environment.

\section{Conclusion}

Together these studies suggest the following:

(1) GABAB inhibits the GBM

(2) For the first time, we proved that baclofen could be used as a drug against GBM

(3) Baclofen takes effect by the GABAB signaling pathway

(4) YAP could promote GMB

(5) Thus, our findings confirm the regulatory relationship between YAP/TAZ and GABA signaling pathway.

\section{Data Availability}

The data used and analyzed during the current study are available from the corresponding author upon request.

\section{Conflicts of Interest}

The authors declare that they have no conflicts of interest.

\section{References}

[1] D. F. Quail and J. A. Joyce, "The microenvironmental landscape of brain tumors," Cancer Cell, vol. 31, no. 3, pp. 326-341, 2017.

[2] A. Omuro and L. M. Deangelis, "Glioblastoma and other malignant gliomas," Journal of the American Medical Association, vol. 310, no. 17, pp. 1842-1850, 2013.

[3] V. Frattini, V. Trifonov, J. M. Chan et al., "The integrated landscape of driver genomic alterations in glioblastoma," Nature Genetics, vol. 45, no. 10, pp. 1141-1149, 2013.

[4] D. N. Louis, H. Ohgaki, O. D. Wiestler et al., "The 2007 WHO classification of tumours of the central nervous system," Acta Neuropathologica, vol. 114, no. 2, pp. 97-109, 2007.

[5] D. Sturm, S. Bender, D. T. W. Jones et al., "Paediatric and adult glioblastoma: multiform (epi)genomic culprits emerge," Nature Reviews Cancer, vol. 14, no. 2, pp. 92-107, 2014.

[6] M. Touat, A. Idbaih, M. Sanson, and K. L. Ligon, "Glioblastoma targeted therapy: updated approaches from recent biological insights," Annals of Oncology, vol. 28, no. 7, pp. 1457-1472, 2017.

[7] H. Ohgaki and P. Kleihues, "Epidemiology and etiology of gliomas," Acta Neuropathologica, vol. 109, no. 1, pp. 93-108, 2005.

[8] A. Tobias, A. Ahmed, K.-S. Moon, and M. S. Lesniak, "The art of gene therapy for glioma: a review of the challenging road to the bedside," Journal of Neurology, Neurosurgery \& Psychiatry, vol. 84, no. 2, pp. 213-222, 2013.

[9] A. Totaro, T. Panciera, and S. Piccolo, "YAP/TAZ upstream signals and downstream responses," Nature Cell Biology, vol. 20, no. 8, pp. 888-899, 2018.

[10] J. Luo and F. X. Yu, "GPCR-hippo signaling in cancer," Cells, vol. 8, no. 5, 2019. 
[11] F. Zanconato, M. Cordenonsi, and S. Piccolo, "YAP/TAZ at the roots of cancer," Cancer Cell, vol. 29, no. 6, pp. 783-803, 2016.

[12] Z. Liu, P. P. Yee, Y. Wei, Z Liu, Y. I Kawasawa, and W Li, "Differential YAP expression in glioma cells induces cell competition and promotes tumorigenesis," Journal of Cell Science, vol. 132, no. 5, 2019.

[13] N. Artinian, C. Cloninger, B. Holmes, A. Benavides-Serrato, T. Bashir, and J. Gera, "Phosphorylation of the Hippo pathway component AMOTL2 by the mTORC2 kinase promotes YAP signaling, resulting in enhanced glioblastoma growth and invasiveness," Journal of Biological Chemistry, vol. 290, no. 32, pp. 19387-19401, 2015.

[14] M. Terunuma, "Diversity of structure and function of GABAB receptors: a complexity of GABAB-mediated signaling," Proceedings of the Japan Academy, Series B, vol. 94, no. 10, pp. $390-411,2018$.

[15] A. Shergalis, A. Bankhead, U. Luesakul, N. Muangsin, and N. Neamati, "Current challenges and opportunities in treating glioblastoma," Pharmacological Reviews, vol. 70, no. 3, pp. 412-445, 2018.

[16] R. Sanchez-Ponce, L.-Q. Wang, W. Lu, J. von Hehn, M. Cherubini, and R. Rush, "Metabolic and pharmacokinetic differentiation of STX209 and racemic baclofen in humans," Metabolites, vol. 2, no. 3, pp. 596-613, 2012.

[17] T. Wang, W. Huang, and F. Chen, "Baclofen, a GABAB receptor agonist, inhibits human hepatocellular carcinoma cell growth in vitro and in vivo," Life Sciences, vol. 82, no. 9-10, pp. 536-541, 2008.

[18] M. Fève, J.-M. Saliou, M. Zeniou et al., "Comparative expression study of the endo-G protein coupled receptor (GPCR) repertoire in human glioblastoma cancer stem-like cells, U87-MG cells and non malignant cells of neural origin unveils new potential therapeutic targets," PLoS One, vol. 9, no. 3, Article ID e91519, 2014.

[19] T. Sun, K. Li, K. Zhu, R. Yan, C. Dang, and D. Yuan, “SNHG6 interacted with miR-325-3p to regulate cisplatin resistance of gastric cancer by targeting GITR," OncoTargets and Therapy, vol. 13, pp. 12181-12193, 2020.

[20] Z. Pei, K.-C. Lee, A. Khan, G. Erisnor, and H.-Y. Wang, "Pathway analysis of glutamate-mediated, calcium-related signaling in glioma progression," Biochemical Pharmacology, vol. 176, p. 113814, 2020.

[21] E. A. El-Habr, L. G. Dubois, F. Burel-Vandenbos et al., "A driver role for GABA metabolism in controlling stem and proliferative cell state through GHB production in glioma," Acta Neuropathologica, vol. 133, no. 4, pp. 645-660, 2017.

[22] C.-L. Da, Y. Xin, and J. Zhao, "Significance and relationship between Yes-associated protein and survivin expression in gastric carcinoma and precancerous lesions," World Journal of Gastroenterology, vol. 15, no. 32, pp. 4055-4061, 2009.

[23] X. Li, L. Yao, Q. Liang, H Qu, and H Cai, "Propofol protects hippocampal neurons from hypoxia-reoxygenation injury by decreasing calcineurin-induced calcium overload and activating YAP signaling," Oxidative medicine and cellular longevity, vol. 2018, Article ID 1725191, 2018.

[24] W. Zhang, Y. Gao, F. Li et al., "YAP promotes malignant progression of lkb1-deficient lung adenocarcinoma through downstream regulation of survivin," Cancer Research, vol. 75, no. 21, pp. 4450-4457, 2015.

[25] J. Cui and W. J. Placzek, "Post-transcriptional regulation of anti-apoptotic BCL2 family members," International Journal of Molecular Sciences, vol. 19, no. 1, 2018.
[26] J. Rosenbluh, D. Nijhawan, A. G. Cox et al., " $\beta$-Catenin-Driven cancers require a YAP1 transcriptional complex for survival and tumorigenesis," Cell, vol. 151, no. 7, pp. 1457-1473, 2012.

[27] Z. Khan, A. A. Khan, H. Yadav, G. B. K. S. Prasad, and P. S. Bisen, "Survivin, a molecular target for therapeutic interventions in squamous cell carcinoma," Cellular and Molecular Biology Letters, vol. 22, no. 1, p. 8, 2017. 